Hydroécol. Appl. (1997) Tome 9 Vol. 1-2, pp. 1-21

\title{
Présentation générale de l'aménagement hydroélectrique de Petit-Saut (Guyane française) et du programme de suivi écologique lié à sa mise en eau
}

\section{General presentation of Petit-Saut hydroelectric installation (French Guiana) and ecological monitoring program}

\author{
C. Sissakian ${ }^{(1)}$ \\ (1) EDF - CNEH - Savoie Technolac - F-73373 Le Bourget-du-Lac
}

Résumé. - Le barrage de Petit-Saut se trouve sur le fleuve Sinnamary en Guyane française.

A la cote de retenue normale (RN $35 \mathrm{~m}$ ), le plan d'eau s'étend sur environ $310 \mathrm{~km}^{2} \mathrm{de}$ forêt (humide, de type amazonien) non déboisée pour un volume total de $3,5.10^{9} \mathrm{~m}^{3}$ (volume utile de $10^{9} \mathrm{~m}^{3}$ entre les cotes $35,00 \mathrm{~m}$ et $31,50 \mathrm{~m}$, niveau minimum d'exploitation). II faut ajouter $110 \mathrm{~km}^{2}$ d'îles et de presqu'îles liées à la topographie vallonnée du site.

En raison principalement de la faible hydraulicité des années 1994 et 1995, et des paliers techniques liés à la surveillance du comportement des ouvrages, le remplissage de la retenue a duré 18 mois. EDF a, en parallèle, étudié et fait réaliser des dispositifs de réoxygénation et de dégazage (ré-aération forcée par diffuseurs d'air comprimé et construction d'un seuil permettant de créer une double cascade dans le chenal de fuite) des eaux turbinées désoxygénées et chargées en gaz réduits.

Pendant toute le durée de remplissage, EDF a mis en œuvre, avec l'aide d'un Comité Scientifique, un programme de suivi écologique qui doit se poursuivre durant les trois premières années de fonctionnement de l'ouvrage, soit jusqu'à mi-1998. Ce programme porte principalement sur la qualité des eaux (chimie et biologie), les modifications hydrologiques, la protection de la faune terrestre (sauvetage, transfert, modifications suite à la fragmentation du milieu), l'évolution structurale et floristique du massif forestier ainsi que les opérations de sauvetage archéologique.

Le programme a nécessité la protection par l'Office National de la Chasse de la retenue, de ses abords et de la zone de relâcher des animaux déplacés.

Mots clés. - Petit-Saut, retenue, remplissage, hydrologie, environnement. 
Abstract. - Petit-Saut dam is located on the Sinnamary river in French Guiana. At full supply level (EI. 35), the reservoir stretches over approximately $310 \mathrm{~km}^{2}$ of wet rain forest left uncleared; the total storage is $350010^{6} \mathrm{~m}^{3}$ (useful storage of $10^{9} \mathrm{~m}^{3}$ between El. 35.00 and 31.50 , the minimum operating level). In addition, there are $110 \mathrm{~km}^{2}$ of islands and peninsulas by reason of the rolling topography of the site.

Mainly because of the poor hydraulic conditions in 1994-95 and the impounding stages required to monitor the structures' behavior, reservoir impounding took 18 months, giving EDF an opportunity to design and build systems to re-oxygenate and degas the oxygen-poor discharge from the turbines with its high reduced gas content (forced re-aeration by compressed air diffusers and construction of a weir to create a double set of falls in the tailrace channel). Throughout the impounding, EDF, with the help of a Scientific Committee, implemented an ecological monitoring program that is expected to continue through the first three years of operation (mid 1995 - mid 1998). This program mainly addresses water quality (chemical and biological aspects), hydrological modifications, protection of land fauna (rescue, transfer, modifications following fragmentation of the environment), structural modifications and changes in the flora in the surrounding forest, as well as operations in view of archaeological preservation.

This program required that the reservoir, its surroundings and the wildlife release area be protected by the National Hunting Agency.

Key words. - Petit-Saut, resenoir, impounding, hydrology, environment.

L'objet de cette communication est de présenter successivement les principales caractéristiques de l'aménagement, puis la spécificité de l'environnement du barrage de PetitSaut [l'impact sur la qualité de l'eau est largement développé par ailleurs dans ce numéro (Richard et al., 1997)], enfin le programme de suivi associé au déroulement de la mise en eau.

\section{PRINCIPALES CARACTÉRISTIQUES DE L'AMÉNAGEMENT}

\section{Le fleuve Sinnamary (fig. 1)}

L'aménagement a été construit sur la rivière Sinnamary. Le Sinnamary prend sa source en plein centre de la Guyane au pied de la montagne
Continent qui culmine à $600 \mathrm{~m}$ environ. II s'écoule en direction du Nord vers l'Atlantique, la longueur de son cours est de $200 \mathrm{~km}$.

La pente moyenne du Sinnamary est très faible, voisine de $0,4 \%$. A l'emplacement du site du barrage, le lit du fleuve est très large, environ $40 \mathrm{~m}$. Les vitesses restent modestes, environ $0,5 \mathrm{~m} / \mathrm{s}$ pour le débit moyen annuel $\left(260 \mathrm{~m}^{3} / \mathrm{s}\right)$. Elles varient de $0,3 \mathrm{~m} / \mathrm{s}$ à $1,5 \mathrm{~m} / \mathrm{s}$. Le lit du Sinnamary comme celui de son principal affluent, le Courcibo, comporte un certain nombre de rapides ou sauts plus ou moins importants.

Le bassin versant au site du barrage (fig. 1)

Le site du barrage au lieu-dit "Petit-Saut" se trouve à $35 \mathrm{~km}$ de la ville de Sinnamary. Le bassin versant au 


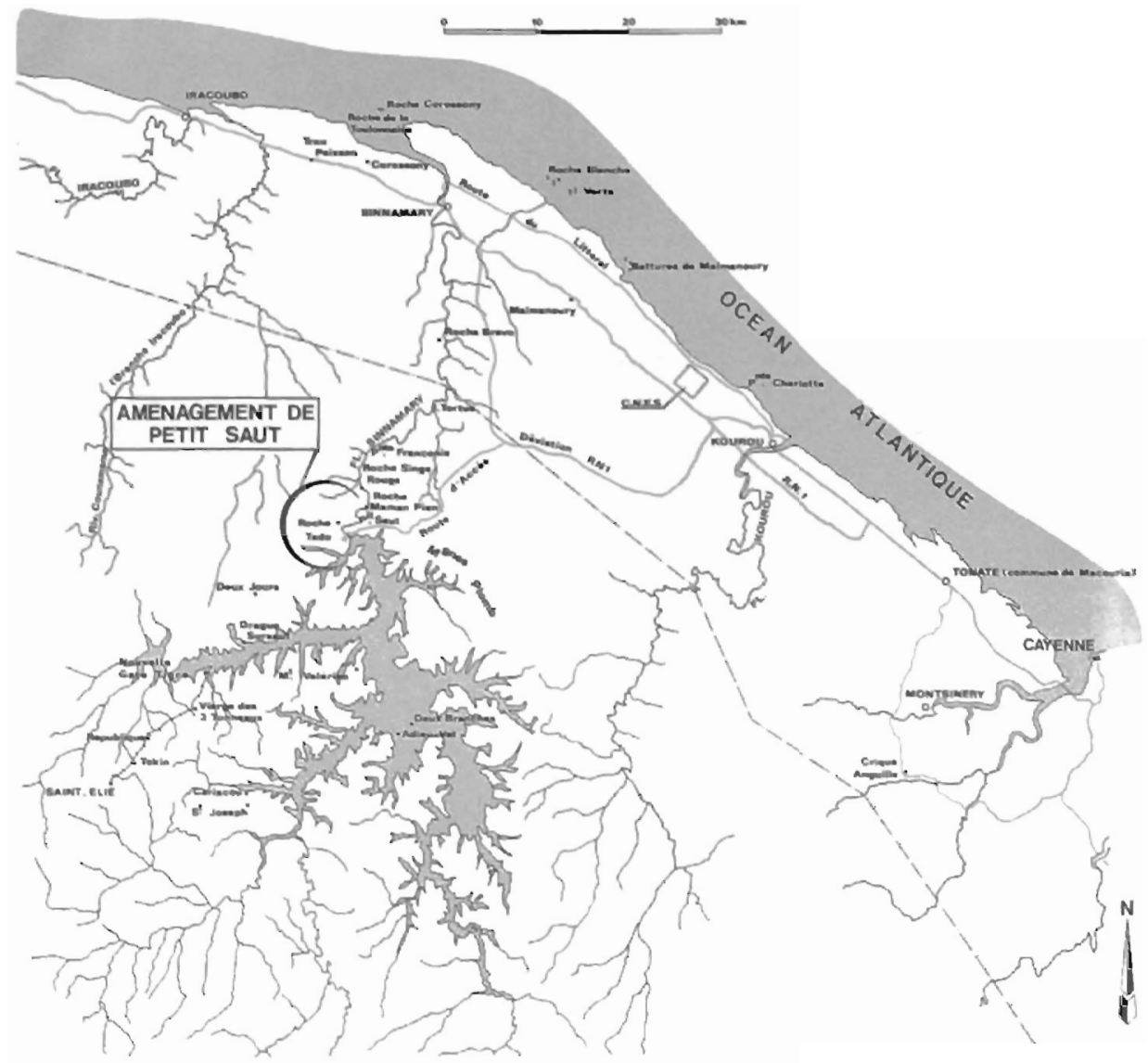

Fig. 1. - Plan de situation

Fig. 1. - Location map

droit du barrage, de forme allongée, est de $6000 \mathrm{~km}^{2}$. Les apports moyens annuels sont de $8,2.10^{9} \mathrm{~m}^{3}$ correspondant à un module de $260 \mathrm{~m}^{3} / \mathrm{s}$. Le débit minimum sur 3 jours consécutifs, avec une fréquence de retour d'une année sur cinq, est de $60 \mathrm{~m}^{3} / \mathrm{s}$. La crue de projet (crue de retour de 10000 ans calculée par la méthode du gradex) est de $3900 \mathrm{~m}^{3} / \mathrm{s}$. Sur les 22 années de mesure, le débit journalier le plus faible enregistré a été de $21 \mathrm{~m}^{3} / \mathrm{s}$ (1995), le plus fort de $1494 \mathrm{~m}^{3} / \mathrm{s}$ (1977). 
Le bassin du Sinnamary reçoit, chaque année, en moyenne $2750 \mathrm{~mm}$ de pluie. Les fortes précipitations et les sols relativement imperméables conduisent à un ruissellement abondant malgré la forte évapotranspiration de la forêt. Le régime climatique est caractérisé en moyenne par une saison des pluies de janvier à juillet, assez fréquemment interrompue par une période de temps plus sec appelée "petit été de mars" bien qu'elle se produise aussi souvent en février qu'en mars, et une saison sèche d'août à décembre.

\section{La retenue (fig. 1)}

Le niveau de retenue normal est de $35 \mathrm{~m}$ correspondant à une surface du plan d'eau de $310 \mathrm{~km}^{2}$ et à une capacité totale de $3,5 \cdot 10^{9} \mathrm{~m}^{3}$. La capacité utile est de $10^{9} \mathrm{~m}^{3}$ et le niveau minimum d'exploitation est de $31,50 \mathrm{~m}$ correspondant à une zone de marnage d'environ $140 \mathrm{~km}^{2}$. A $35 \mathrm{~m}$, $110 \mathrm{~km}^{2}$ d'îles et de presqu'îles ont été créées par la mise en eau en raison de la topographie vallonnée du site (relief dit "en peau d'orange"). Ces valeurs sont, actuellement en cours de vérification à l'aide d'images satellite. La retenue n'a pas été déboisée avant la mise en eau sauf à proximité du barrage.

\section{Les ouvrages (fig. 2)}

Le barrage a été réalisé en béton compacté au rouleau non zoné avec un masque amont d'étanchéité en béton traditionnel. La crête, de développement $740 \mathrm{~m}$, est à la cote $37 \mathrm{~m}$ avec une hauteur sur fondation de $47 \mathrm{~m}$. L'usine extérieure en béton tra- ditionnel est intégrée au barrage (barrage de prise / usine). Elle est équipée de 4 turbines Kaplan à axe vertical au débouché des 4 conduites forcées de $4,70 \mathrm{~m}$ de diamètre et de $26 \mathrm{~m}$ de long traversant le barrage, correspondant aux 4 prises d'eau. La puissance installée est de $116 \mathrm{MW}$ et le productible moyen annuel est de 560 GWh. L'équipement est complété par des dispositifs d'évacuation de crues : un clapet de surface et un déversoir de surface permettent d'évacuer respectivement un débit de $340 \mathrm{~m}^{3} / \mathrm{s}$ et de $200 \mathrm{~m}^{3} / \mathrm{s}$ sous $36,50 \mathrm{~m}$, un évacuateur de fond composé de 3 pertuis de $7 \mathrm{~m}$ sur $7 \mathrm{~m}$ permet d'évacuer $3045 \mathrm{~m}^{3} / \mathrm{s}$ sous $36,50 \mathrm{~m}$.

Les eaux turbinées sont rejetées dans le Sinnamary à l'aval des ouvrages par l'intermédiaire d'un canal de fuite de $430 \mathrm{~m}$ de long et de $80 \mathrm{~m}$ de large.

\section{DISPOSITIONS PARTICULIĖRES LIÉES À LA QUALITÉ DE L'EAU}

\subsection{Rappel des prévisions de qualité d'eau}

Les enseignements recueillis sur des sites de barrage en forêt primaire tropicale et les résultats d'un modèle mathématique de simulation (EDF, 1987) mettaient en évidence, dans la retenue, deux zones: une masse d'eau inférieure, l'hypolimnion, anoxi- 


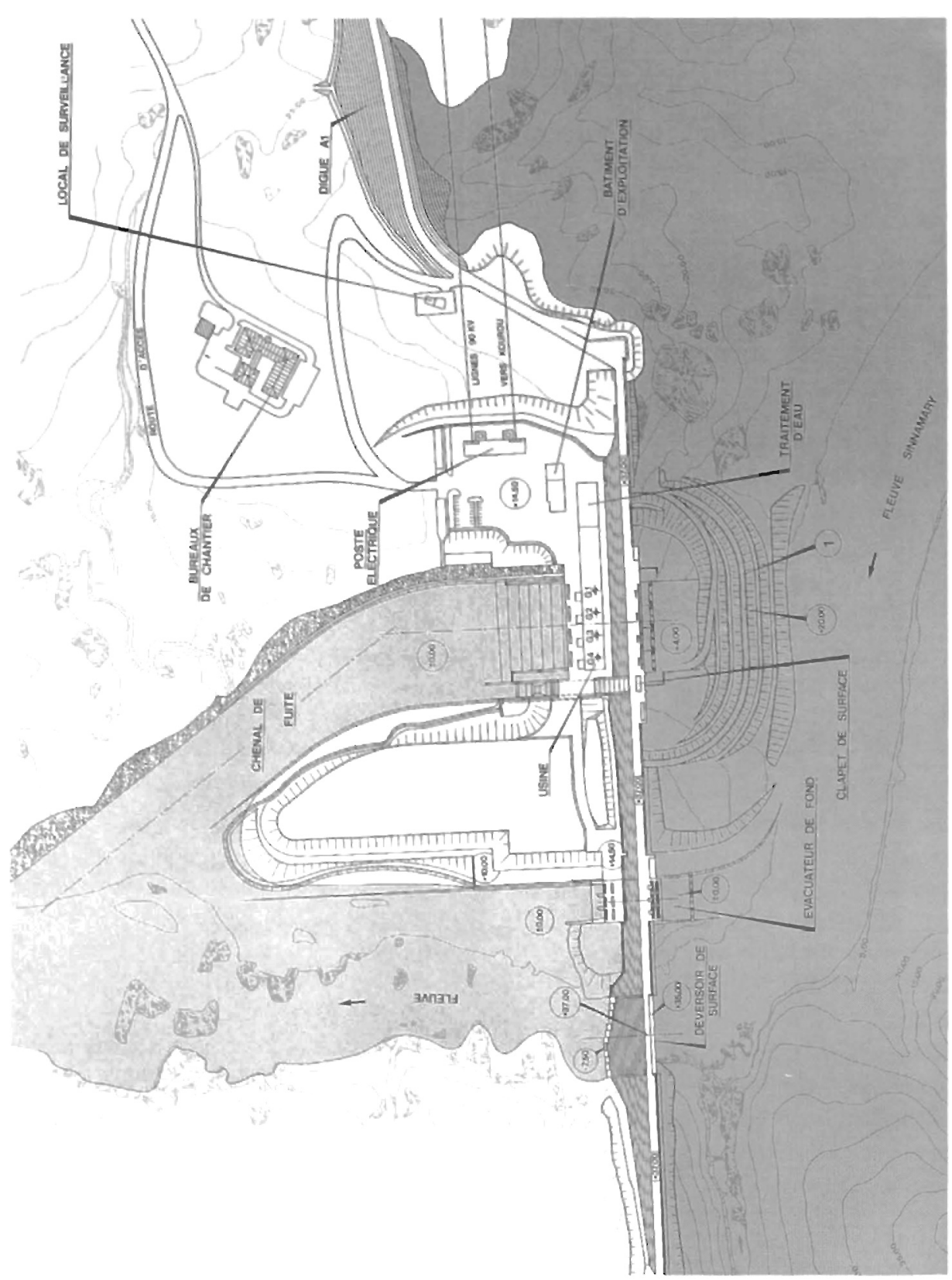

Fig. 2. - Plan général de l'aménagement

Fig. 2. - General layout of the scheme 
que, et une couche supérieure de quelques mètres, l'épilimnion, bien oxygénée ainsi que des risques de désoxygénation pour le cours d'eau à l'aval des ouvrages. L'amélioration dans le temps de la qualité de l'eau de la retenue (épuisement progressif de la matière à dégrader) devait permettre au bout de quelques années le retour à de meilleures conditions.

En accord avec les pouvoirs publics, le choix de privilégier, dans la mesure du possible, l'aval du Sinnamary a été fait. Des mesures ont donc été prises pour améliorer la qualité de l'eau de ce tronçon plus particulièrement pendant les premiers mois prévus comme les plus "sensibles" et, à plus long terme, pour turbiner les eaux de surface de meilleure qualité.

\subsection{Dispositions retenues (fig. 2)}

En fonction de ce qui vient d'être décrit plus haut, des dispositions spéciales ont été retenues pour les contraintes de qualité d'eau (Gosse et al., 1997) :

- passage du débit réservé pendant le remplissage par les évacuateurs de fond dont l'équipement adapté a permis de créer d'importants remous entrainant la réoxygénation des eaux et le dégazage des gaz réduits.

Des diffuseurs d'air comprimé situés à l'amont immédiat des évacuateurs dans la retenue n'ont pas eu d'effet significatif sur la qualité de l'eau.
- réalisation d'une digue à l'amont des prises d'eau et à une cote de crête de $20 \mathrm{~m}$ qui ne semble pas avoir d'effet significatif sur la qualité de l'eau à l'aval.

\subsection{Rappel sur le choix du débit réservé}

En raison de la configuration de l'aménagement, des caractéristiques hydrologiques du Sinnamary, de l'éventuelle incidence sur le coin salé et des résultats du modèle mathématique de prévision de qualité d'eau (optimum entre la qualité de l'eau dans la retenue et à l'aval), il a été décidé de laisser en permanence à l'aval des ouvrages un débit de $100 \mathrm{~m}^{3} / \mathrm{s}$ pendant le remplissage et $80 \mathrm{~m}^{3} / \mathrm{s}$ en exploitation. La valeur de $80 \mathrm{~m}^{3} / \mathrm{s}$ correspond, à peu près, à la valeur minimale turbinable en exploitation.

\section{PLANNING GÉNÉRAL (tab. 1)}

L'étude d'impact a débuté en 1983. L'enquête publique a eu lieu en novembre 1987. Le Décret de Concession et de Déclaration d'Utilité Publique a été signé le 18 mai 1989.

Le remplissage a débuté en janvier 1994, juste après l'autorisation préfectorale de remplissage accordée le 5 janvier 1994.

Les principaux travaux de Génie Civil ont commencé mi-1989 pour se 
Tableau 1. - Petit-Saut: planning général

Table 1. - Petit-Saut: general work schedule

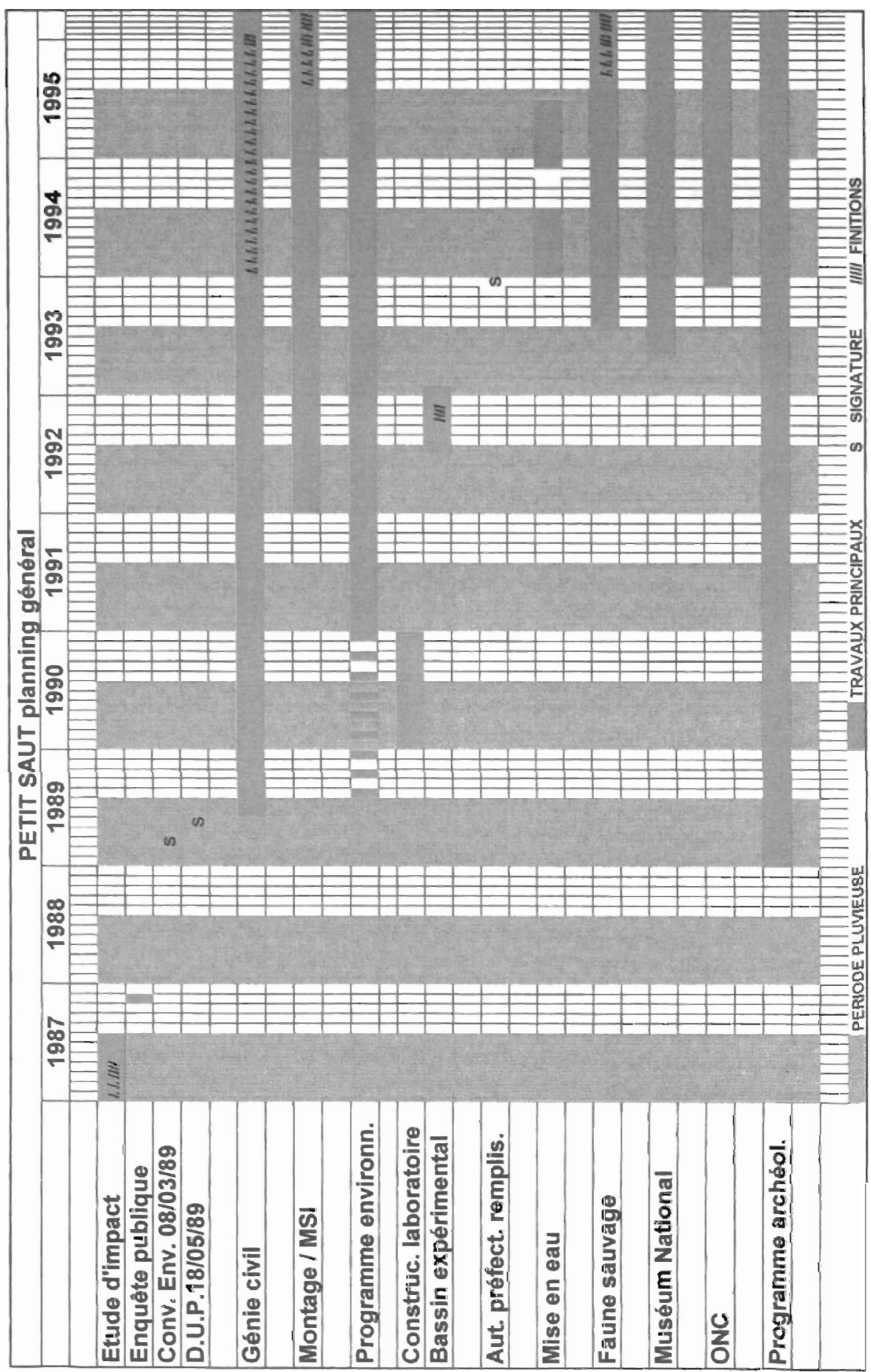


terminer fin 1993. Les travaux relatifs à l'équipement électromécanique ont commencé début 1992 pour se terminer en juillet 1995.

\section{PROGRAMME DE SUIVI ÉCOLOGIQUE MIS EN PLACE POUR LE REMPLISSAGE DE LA RETENUE ET LES PREMIËRES ANNÉES D'EXPLOITATION}

\subsection{Historique}

Ce programme a été établi à partir :

- des conclusions de l'étude d'impact (1987),

- des engagements d'EDF listés dans la Convention "Environnement" du 8 mars 1989 annexée au décret de Concession et de Déclaration d'Utilité Publique de l'aménagement hydroélectrique de Petit-Saut,

- des avis du Comité Scientifique mis en place par EDF pour conseiller l'Entreprise dans la réalisation de ses engagements,

- des enseignements tirés des barrages réalisés en forêt tropicale,

- du retour d'expérience des suivis réalisés en métropole.

\subsection{Présentation du programme (tab. 2)}

Conformément donc aux actions prioritaires listées dans la Convention Environnement, un programme a été mené dès 1989 sur le site. Cependant, l'essentiel des actions a commencé en janvier 1991 (date de fin de construction du laboratoire "en- vironnement "sur le site) et est présenté ci-après.

La plupart des organismes scientifiques impliqués dans le programme interviennent sur le projet de PetitSaut depuis 1983, date de début de réalisation de l'étude d'impact, ou ont èté choisis avec l'aide du Comité scientifique.

Le programme suivi à partir de Janvier 1991 et renforcé mi-1993 peut être scindé en trois grands volets.

- le volet aquatique comprenant l'étude physico-chimique (dont l'évolution du mercure) et biologique de la retenue et du tronçon aval du Sinnamary, réalisée sur place par le laboratoire de site géré par HYDRECO ainsi que l'étude ichtyologique (ORSTOM), celle des échanges gazeux avec l'atmosphère (Université de Toulouse), le suivi de la végétation aquatique (Université de Savoie / ESIGEC) et le suivi des biomasses algales (CNRS Montpellier),

- le programme de protection de la faune terrestre consistant en:

- un plan de sauvetage et de suivi des animaux déplacés vers une zone de relâcher (translocation) mené par une équipe de vétérinaires et de biologistes,

- une étude des modifications écologiques et comportementales de la faune terrestre suite à la fragmentation du milieu, menée par le Muséum National d'Histoire Naturelle,

- un ensemble d'autres actions comprenant : 
Tableau 2. - Liste des organismes de recherches intervenant dans le programme Environnement et principales études réalisées.

Table 2. - Environmental program, Studies and institutes in charge.

\section{Etudes et intervenants}

\section{Orstom Cayenne}

Suivi des débits

Évolution du coin salé

Suivi de la retenue et évolution de l'estuaire par télédétection

Suivi ichtyologique amont et aval

Partenariat: Maintien de la diversité d'un peuplement de poissons (Université Claude-Bernard de Lyon)

Évolution floristique et structurale du massif forestier

Laboratoire hydrobiologie de Petit-Saut (Hydreco)

Étude hydrochimique et hydrobiologique (retenue et rivière)

Université de Toulouse

Echanges gazeux biosphère/atmosphère

Bilan des respirations anaérobies

CNRS Montpellier (Hydreco)

Algues

Partenariat: Programme spécifique lié à la dégradation de la M.O. (Montpellier 2/Aix-Marseille 2/U. Perpignan)

Universitè de Savoie (ESIGEC)

Végétation flottanle

Muséum national d'histoire naturelle

Modifications écologiques et comportementales de la faune terrestre

Fragmentation

Régénération forestière

Programme Faune Sauvage

Plan de sauvetage et suivi des animaux relâchés

Divers partenariats scientifiques

(Ecole vétérinaire de Toulouse)

Universitè de Montpellier 2

Suivi de deux especes de paresseux

Génétique des mammiféres de la forêt guyanaise

Institut Pasteur de Cayenne

Étude des associations hôtes vecteurs et hôtes agents pathogènes

Association pour les fouilles archéologiques nationales

Programme archéologique de Petit-Saut

- le suivi des débits, le suivi de la retenue et de l'estuaire par télédétection (ORSTOM),

- l'étude de l'évolution structurale et floristique du massif forestier (ORSTOM),
- la mise en œuvre d'un programme archéologique (AFAN).

Le programme a nécessité la protection de la retenue, de ses abords et de la zone de relâcher par l'Office National de la Chasse. Ces actions de protection ont été entièrement financées par EDF. 
Tableau 3. - Petit-Saut; planning de mise en eau.

Table 3. - Petit-Saut: impounding schedule.

\begin{tabular}{|c|c|c|c|c|c|}
\hline \multicolumn{6}{|c|}{ PETIT SAUT planning mise en eau } \\
\hline & 1993 & \multicolumn{2}{|c|}{1994} & \multicolumn{2}{|c|}{1995} \\
\hline & & $\square$ & & & \\
\hline \multicolumn{6}{|l|}{ Génie civil } \\
\hline \multirow{2}{*}{\multicolumn{6}{|c|}{ Montage / MSI }} \\
\hline & & & & & \\
\hline \multicolumn{6}{|l|}{ Programme environn. } \\
\hline \multirow{2}{*}{\multicolumn{6}{|c|}{ Aut. préfect. remplis. }} \\
\hline & & & & & \\
\hline \multicolumn{6}{|l|}{ Mise en eau } \\
\hline \multicolumn{6}{|l|}{ Turbinage } \\
\hline \multirow{2}{*}{\multicolumn{6}{|c|}{ Seuils de réoxygénation }} \\
\hline & & & & & \\
\hline \multicolumn{6}{|l|}{ Etudes } \\
\hline \multicolumn{6}{|l|}{ Construction } \\
\hline \multicolumn{6}{|l|}{ Essais } \\
\hline \multicolumn{6}{|l|}{ Faune sauvage } \\
\hline \multicolumn{6}{|l|}{\begin{tabular}{|l|} 
Muséum National \\
\end{tabular}} \\
\hline & & & & & \\
\hline \multicolumn{6}{|l|}{ ONC } \\
\hline \multicolumn{6}{|l|}{ Programme archéol. } \\
\hline & & & & & \\
\hline & & DE PLUVIEUSE & & $\frac{1}{1}$ & \\
\hline & S SIGNATURE & 11111 & IMI FINITIONS & 11011 & \\
\hline
\end{tabular}

5 DÉROULEMENT DE LA MISE EN EAU DU BARRAGE (tab. 3)

\subsection{Planning}

L'autorisation préfectorale de mise en eau a été donnée le 5 janvier 1994. La mise en eau de la retenue a commencé le 6 janvier 1994 et s'est terminée le 28 juillet 1995. Cette mise en eau s'est déroulée en plusieurs phases:

- phase $n^{\circ} 1$ jusqu'à la cote $31 \mathrm{~m}$ atteinte le 8 juillet 1994 avec un palier de deux semaines (du 14 au 27 février 1994) à la cote $19 \mathrm{~m}$, 
- phase $n^{0} 2$ correspondant à un arrêt de remplissage à la cote $31 \mathrm{~m}$ du 8 juillet au 9 décembre 1994,

- phase $n^{\circ} 3$ de la cote $31 \mathrm{~m}$ à la cote $35 \mathrm{~m}$ (en fait $34,95 \mathrm{~m}$ ) atteinte le 14 juin 1995,

- phase $n^{0} 4$ correspondant au palier technique de fin de mise en eau à la cote $35 \mathrm{~m}$ du 14 juin au 28 juillet 1995.

\subsection{Allongement de la durée de la mise en eau}

La durée de cette mise en eau plus longue que prévue initialement (environ 6 à 9 mois en année moyenne) - est liée à divers événements. Nous retiendrons principalement une pluviométrie plutôt faible à partir d'avril 1995, ou "mal placée" par rapport aux paliers techniques prévus en fonction d'une hydraulicité moyenne. A la fin du palier technique, à $31 \mathrm{~m}$ (5 août 1994) et donc au début de la saison sèche, il a été décidé d'arrêter la mise en eau jusqu'à la prochaine saison des pluies. En effet, les simulations hydrologiques (débit réservé $100 \mathrm{~m}^{3} / \mathrm{s}$, débit moyen saison sèche: $\quad 163 \mathrm{~m}^{3} / \mathrm{s}$ ) incluant les contraintes techniques montraient que le remplissage complet de la retenue nécessitait, à un ou deux mois près, les apports de cette future saison des pluies. Cet arrêt a permis de continuer les essais des groupes dans la limite des débits entrants tout en ne maintenant qu'une surveillance technique allégée des ouvrages.
En parallèle, pendant les premiers mois du remplissage, le suivi de la qualité de l'eau avait très vite mis en évidence un phénomène de désoxygénation du cours aval du Sinnamary plus important que prévu.

En effet, les premiers essais de turbinage réalisés lorsque la cote $25 \mathrm{~m}$ a été atteinte ont montré que pour maintenir une teneur en oxygène dissous, à l'aval des ouvrages, supérieure ou égale à $2 \mathrm{mg} / \mathrm{l}$, il était nécessaire d'évacuer de l'eau par les évacuateurs de fond dans le rapport : $25 \%$ turbinés et $75 \%$ évacués.

L'arrêt de la mise en eau a donc aussi permis d'étudier et de mettre en place dans le canal de fuite de l'usine un seuil oxygénant permettant de réoxygéner et de dégazer les eaux turbinées (cf. Gosse et al., 1997).

\section{Les dispositifs de réoxygénation} (fig. 3)

Les études de conception du seuil ont eu lieu en liaison avec le département Environnement de la DER et un modèle physique a été réalisé par le Laboratoire National d'Hydraulique d'EDF. Les études ont débouché sur la réalisation d'un seuil de forme complexe (cellules en nid d'abeilles) en acier, ancré dans un radier en béton, à $130 \mathrm{~m}$ environ de l'usine dans le canal de fuite. Cette conception repose sur l'augmentation de la longueur de crête de déversement, une diminution de la hauteur de la lame déversante, la création de deux chutes de deux mètres environ chacune ainsi que de fosses de dissipa- 


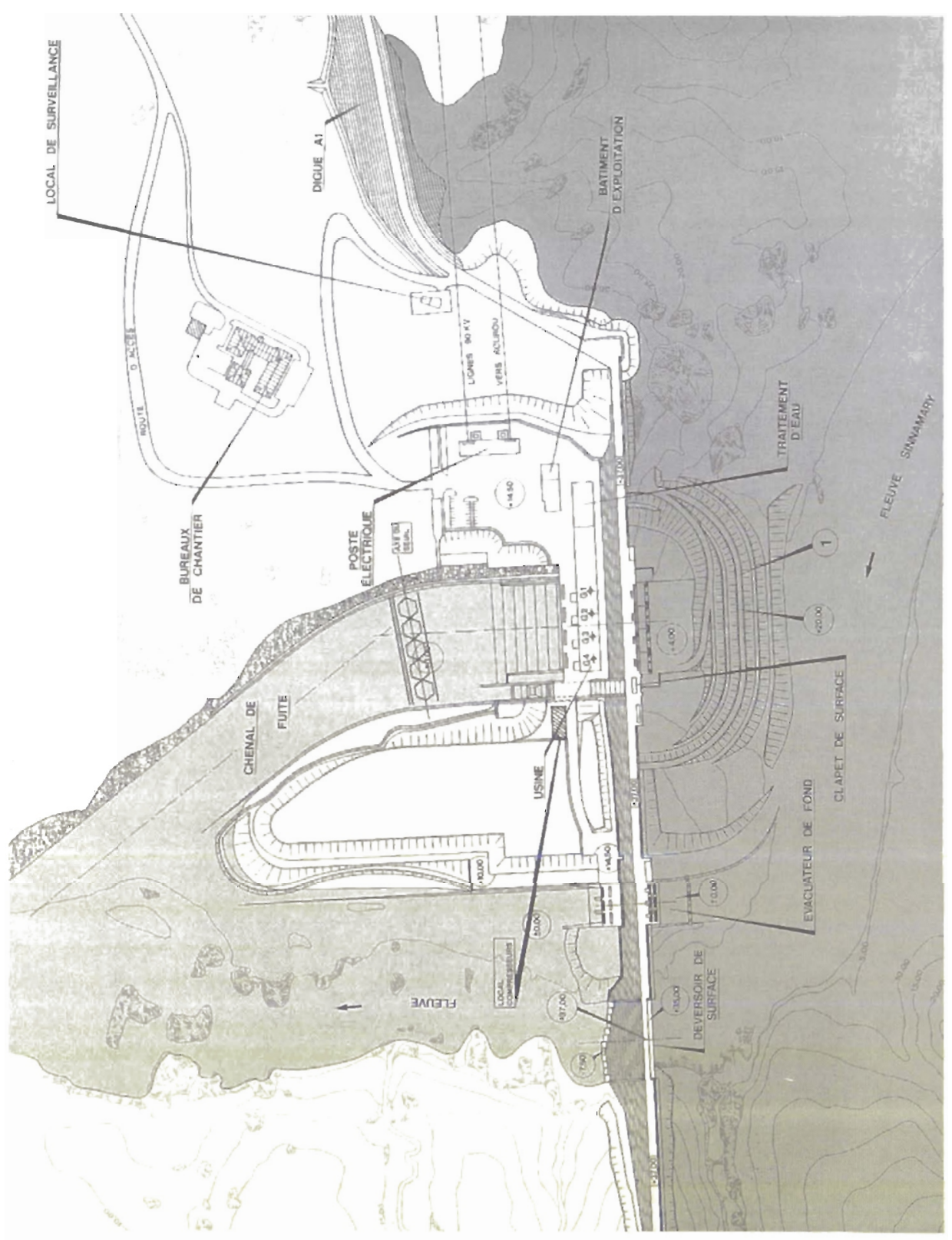

Fig. 3. - Plan général de l'aménagement incluant les dispositif́s de réoxygénation.

Fig. 3. - General layout of the scheme including systems to re-oxygenate. 
tion permettant un bon brassage de l'eau (Gosse et al., 1997).

En complément au seuil déversant métallique, un dispositif de réoxygénation forcée a aussi été installé dans le canal de fuite à l'aval immédiat de l'usine. Les essais ont par la suite montré que l'effet de la réoxygénation forcée était masqué par l'effet prépondérant du seuil.

Etudiés à partir de juillet 1994, décidés fin septembre 1994, construits entre décembre 1994 et février 1995, ces dispositifs ont permis la reprise du turbinage le 2 mars 1995 tout en maintenant une oxygénation convenable tout le long du Sinnamary.

\section{La pluviométrie (Fig. 4)}

La phase $n^{0} 1$ se caractérise par une pluviométrie d'abord élevée en janvier et février mais les contraintes techniques de montée des eaux ne permettent pas de stocker tous les apports. Ces contraintes associées à une plus faible hydraulicité à partir de mars 1994 n'ont permis d'atteindre la cote $31 \mathrm{~m}$ qu'à l'approche de la saison sèche.

La phase $n^{\circ} 2$ qui correspond à l'arrêt du remplissage à la cote $31 \mathrm{~m}$ présente par contre une hydraulicité plus élevée que la moyenne. Elle permettra donc, malgré les problèmes de désoxygénation, un certain nombre d'essai des machines.
La reprise du remplissage lors de la phase $n^{\circ} 3$ se caractérise par une hydraulicité très faible à l'exception du mois de mars 1995. Le mois de mars correspond à la date de reprise du turbinage qui avait été arrêté en décembre pour la construction du seuil déversant. Ce n'est qu'en juin 1995, mois où les débits entrants retrouvent une valeur équivalente à la moyenne, que la cote $35 \mathrm{~m}$ sera atteinte.

\section{6 ÉVOLUTION DE LA QUALITÉ DE L'EAU}

L'évolution de la qualité de l'eau et plus particulièrement les problèmes de désoxygénation sont déjà évoqués ci-dessus. Ils font aussi l'objet d'articles de ce numéro.

Nous rappellerons simplement les principales étapes.

Dans la retenue on a assisté rapidement à la mise en place de la stratification de la masse d'eau avec apparition d'une thermocline et d'une oxycline. Le milieu s'est enrichi en sels minéraux, matière organique, métaux et gaz réduits dissous. Un gradient de la surface au fond s'est mis en place et s'est maintenu. L'épilimnion s'est épaissi progressivement jusqu'à 5 mètres environ. Cette valeur reste relativement stable.

Les processus intervenant pendant la phase de remplissage n'ont pas été uniquement commandés par un sim- 


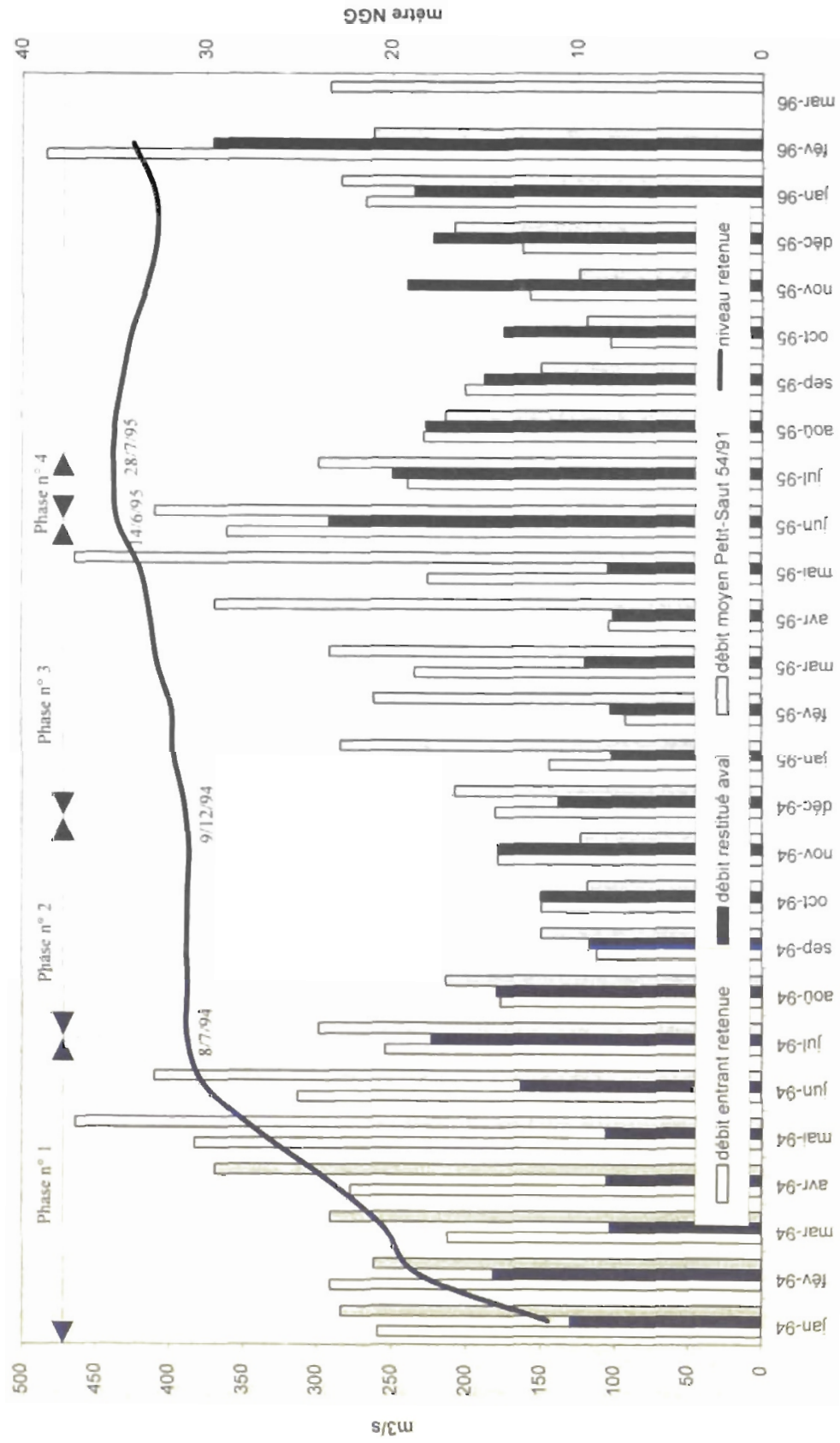

Fig. 4. - Remplissage de la retenue de Petit-Saut.

Fig. 4. - Petit-Saut reservoir impounding. 
ple rapport entre une phase liquide (les apports) et une phase réactive (la matière noyée), mais on a montré que le temps de renouvellement des eaux (crues ou saison sèche, évacuation importante par les pertuis de fond / turbinage ou stockage) influençait la nature et l'intensité des réactions.

Au cours de la première année de mise en eau, le stock de méthane dissous a augmenté régulièrement ainsi que les émissions à la surface du lac. On a ensuite assisté à une stabilisation puis à une tendance à la diminution. Au bout d'un peu plus d'un an, les émissions de méthane à la surface du lac se sont pratiquement arrêtées.

Une explication est la présence de bactéries méthanotrophes qui vivent en milieu oxygéné. Sur ce point deux hypothèses, de nature différente et peut-être complémentaire, restent à préciser. La première (Dumestre et al., 1997) est que ces bactéries ont pu se développer lorsqu'il y a eu diminution du flux d'hydrogène sulfuré (toxique pour de nombreux organismes) provenant de la matière organique du fond et intercepté par les bactéries du cycle du souffre localisées sous l'oxycline. Cette hypothèse pourra être confirmée lorsque ces bactéries pourront être mise en évidence de façon directe.

La deuxième (Gosse et al., à paraître) est que ces bactéries ont pu se développer sur un volume de plus en plus grand en surface du lac (l'oxycline a gagné 1 mètre de mi-94 à début 95) facilitant leur activité concentrée près de la thermocline.
Plusieurs facteurs peuvent expliquer l'épaississement de la thermocline, comme la photosynthèse ou la diminution de puits d'oxygène non liés au méthane (Richard et al., 1997 Gosse et al., 1997).

Les analyses de mercure (le mercure est lié aux activités d'orpaillage) n'ont pas montré jusqu'à maintenant d'augmentation de teneurs dans la chair des poissons. On enregistre plutôt une tendance à la baisse.

A l'aval, la qualité de l'eau, à l'amont du seuil de réoxygénation et de dégazage, correspond à celle de la retenue. Le dégazage artificiel sur le seuil entraîne des émissions importantes de méthane à l'atmosphère. Mais comme on l'a vu au paragraphe 5.2., la bonne efficacité du seuil en matière de réoxygénation et de dégazage permet de maintenir une oxygénation correcte jusqu'à l'estuaire.

\section{PROGRAMME DE PROTECTION DE LA FAUNE TERRESTRE (tab. 4)}

Les actions relatives à la protection de la faune terrestre ont fait l'objet de deux programmes: le programme Faune Sauvage explicité dans ce paragraphe et le programme Muséum qui fait l'objet du paragraphe suivant.

Le programme Faune Sauvage a été confié à une équipe de six personnes formée de vétérinaires et de biologistes sous la direction d'un vétérinaire spécialiste de la faune sau- 
Tableau 4. - Programme "Faune sauvage" - Évolution des captures. Table 4. - "Wild Fauna" program - Captures progress.

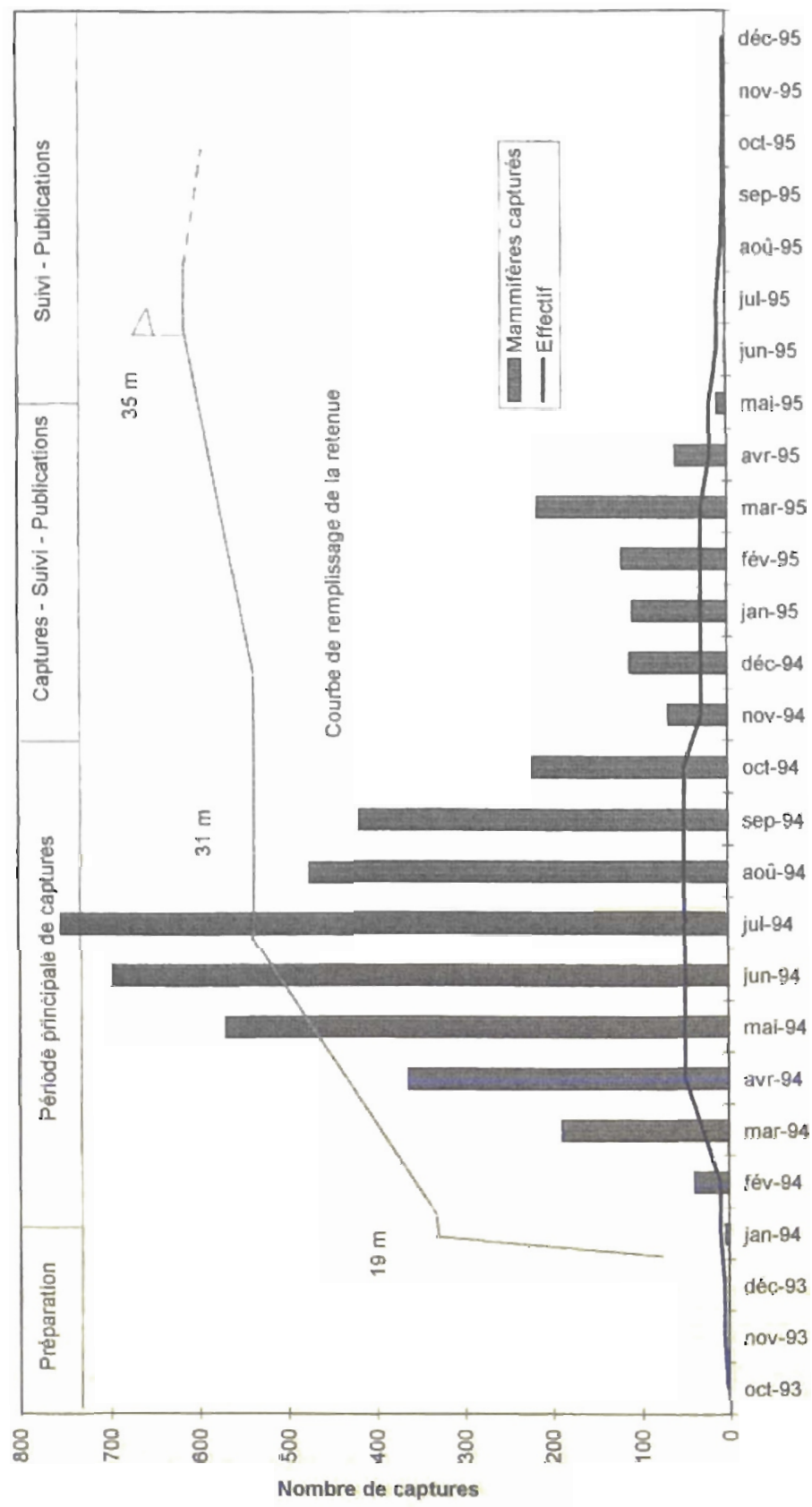


vage. Une douzaine d'étudiants vétérinaires ont aussi participé aux opérations et un agent EDF-CNEH a eu en charge l'ensemble des problèmes de logistique. La structure comprenait aussi une secrétaire. Les opérations de capture, transport, layonnage, entretien et ravitaillement ont été assurées par des entreprises locales qui ont employé jusqu'à une quarantaine d'ouvriers.

La structure était composée d'une base générale à Petit-Saut qui comprenait la base-vie, un centre de capture, le centre de transit et l'hôpital vétérinaire. L'autre centre de capture ainsi qu'une seconde base-vie se trouvaient vers le milieu de la retenue à Saut Vata. La zone de relâcher qui jouxte la retenue a été équipée de carbets de passage.

Pour des raisons de sécurité, tous les centres et la zone de relâcher ont été équipés de relais radio.

La phase de préparation a commencé en octobre 1993. Par la suite, on peut distinguer 3 périodes de capture liées à l'évolution de la mise en eau :

- 03/01/94 à 14/02/94: c'est la période de montée des eaux la plus rapide mais la surface couverte est faible. Aucune opération de capture n'a été entreprise (réception du matériel, essais préliminaires,...),

- 14/02/94 à 30/10/94: c'est la période principale de capture. Jusqu'à fin juin, $25 \%$ du temps de travail a aussi consisté en un travail primordial de prospection et de reconnaissances de terrain (îles, accès, indices de pré- sence...) et les captures ont eu lieu principalement par piégeages. A partir de cette date, plus de $50 \%$ de l'effort de capture a été dirigé vers les mammifères arboricoles,

- 31/10/94 à 28/05/95 : l'effectif des équipes de capture a été réduit de $50 \%$.

Un total de 3202 mammifères, 186 oiseaux, 1386 tortues terrestres et 374 serpents, a été capturé sur la retenue soit 5193 animaux, auquel viennent s'ajouter 76 autres mammifères et 425 serpents trouvés sur la route de Petit-Saut. De façon plus anecdotique, les captures ont aussi porté, par exemple, sur des tortues aquatiques, des mygales, des crapauds-buffles, des cailmans ou des lézards.

En tout, plus de 5800 animaux ont été capturés et ont transité par le centre vétérinaire.

Des données (lieu de capture, sexe, poids, taille, résultats de l'examen clinique) et/ou du matériel biologique (sang, biopsie, parasites, venin) ont été collectés sur l'ensemble des animaux capturés. L'exploitation de ces données est en cours et le matériel biologique utilisé et valorisé par la communauté scientifique. II faut mentionner la découverte d'une nouvelle espèce de rongeur pour la science, Isothrix sinnamariensis (Vié et al., 1996) et de quatre nouvelles espèces de serpent pour la Guyane (Vidal et al., in press).

Le suivi éco-éthologique des animaux déplacés dans la zone de relâcher se poursuit et la très grande 
majorité de ces animaux semble survivre.

Associés à des techniques modernes (études génétiques en cours), ces travaux contribuent à une meilleure connaissance de la faune guyanaise.

Dans la retenue, très peu d'animaux morts ont été vus sur les zones accessibles. Outre l'efficacité du plan de sauvetage, il faut signaler également les capacités naturelles de fuite et d'adaptation des animaux. Actuellement, cependant, on peut voir de place en place un "surpeuplement" des berges et les animaux semblent moins farouches dans certaines zones visitées régulièrement par les chercheurs du Muséum. Ces mêmes chercheurs ont signalé la présence récente d'animaux morts sur certaines îles de faibles dimensions.

\section{PROGRAMME DU MUSEUM NATIONAL D'HISTOIRE NATURELLE}

L'étude porte sur les effets de la fragmentation de l'écosystème sur la faune sauvage de vertébrés. Elle est menée sur plusieurs sites insulaires et une zone témoin sur la terre ferme. Le fonctionnement en archipel de certaines îles constitue un modèle intéressant pour tester les flux de population. Ces sites se situent sur la branche Courcibo de la retenue.

L'étude du cadre initial a montré que la zone était peuplée par une faune de vertébrés classique et complète de la forêt dense humide guyanaise, sans espèces endémiques. Pour la flore, certaines plantes rares ailleurs étaient très abondantes à Saint-Eugène (camp du Muséum).

L'étude des effets attendus porte sur les points suivants :

- Modification de la richesse spécifique? Fuite?

- Modification du sceptre d'espèces? Qui disparaît? Qui s'introduit dans le peuplement?

- Modification de la hiérarchie au sein des peuplements? Des espèces favorisées?

- Des resserrements de domaines vitaux, de territoires?

- Des modifications de niches écologiques, de relations sociales au sein d'un peuplement?

- Une dérive génétique sur les petits rongeurs?

- En quoi l'impact sur les vertébrés se répercute-t-il sur la régénération forestière?

La plupart de ces études font l'objet de thèses qui seront soutenues d'ici fin 1998. Comme les études menées sur la faune déplacée et en cohérence avec elles, elles permettront d'avoir une meilleure connaissance de la faune guyanaise.

\section{PROTECTION DE LA ZONE : CONVENTION EDF/ONC}

La protection de la retenue, de ses abords et de la zone de relâcher a fait l'objet d'une convention EDF/ONC de novembre 1993 à juillet 1996, pro- 
longation de six mois incluse. Dans ce cadre trois gardes nationaux de la chasse et de la faune sauvage de l'ONC ont constitué la première brigade mobile en Guyane (B.M.I. GUYANE). Les objectifs de la convention rappelés ci-dessous ont été largement atteints :

\section{blic}

- prévention et information du pu-

- actions techniques relatives aux techniques de piégeage, à l'aide aux captures et à la participation au suivi des animaux relâchés

- actions de contrôle relatives à la chasse et à la circulation et à l'orpaillage.

Bien que la zone principale d'intervention de la B.M.I. ait été le site du barrage, les gardes ont été sollicités pour des interventions hors zone par les différents services de contrôles guyanais.

Depuis la fin de la convention avec $E D F$, une brigade mixte, ONF/ONC, s'est substituée à la B.M.I. afin de pérenniser les contrôles relatifs à la protection de la nature en Guyane.

Une réflexion sur un statut de "réserve naturelle volontaire" pour la zone de Petit-Saut est actuellement menée par les pouvoirs publics concernés.

\section{MASSIF FORESTIER}

L'évolution structurale et floristique du massif forestier a été suivie pendant le remplissage de la retenue.
L'étude a d'abord été menée sur l'état initial de la connaissance taxonomique du bassin du Sinnamary puis sur les caractéristiques structurales, floristiques ou stratégiques qui définissent le mieux la relation végétation-eau.

La végétation a disparu progressivement dans la zone inondée pendant les 18 mois de remplissage.

Les individus les plus sensibles ont été les lianes, très vite effeuillées et les arbres les plus communs présents sur les anciennes rives. Pour la majorité des arbres le stress s'est traduit par différents changements : floraison hâtive, "flot " de jeunes feuilles. Seuls quelques rares individus ont supporté ce stress plus de dix-huit mois. Actuellement il ne reste que des arbres morts portant de place en place des épiphytes ou des hémi-épiphytes. La disparition totale des arbres suivra un processus de désagrégation progressive depuis les pousses jusqu'aux troncs. L'expérience a montré que cette disparition était à deux vitesses : deux à cinq ans pour la couronne et vingt à cinquante ans ou plus pour les troncs.

La végétation des nombreuses îles qui maintenant parsèment la retenue n'a pas enregistré de modifications notables.

Mais on peut penser que la reconstruction de l'ensemble des berges se fera à une autre échelle de temps en fonction des pentes, de la fréquence de marnage et des espèces aptes à coloniser les milieux inondables. 


\section{Insectes}

Au sein de la biocénose terrestre, les insectes représentent la premiere biodiversité et en milieu tropical certainement la première biomasse. Compte tenu de leur richesse spécifique en Guyane, les insectes ont été étudiés au travers de quelques familles de lépidoptères (en particulier les sphingidae), de coléoptères (en particulier les cérambycidae) ou d'odonates. De nombreuses espèces nouvelles pour la science ont été répertoriées lors des études réalisées par le laboratoire de site et l'ORSTOM en collaboration avec de nombreux autres organismes (Museum, universités,...).

\section{PROGRAMME ARCHÉOLOGIQUE}

L'opération menée sur le site de Petit-Saut entre 1989 et 1996 est la première opération archéologique d'envergure en Guyane. Elle a prouvé qu'une approche innovante pouvait être très fructueuse. Le programme archéologique de Petit-Saut constitue aussi la première opération de sauvetage dans un département d'outremer. Sa réalisation a été confiée à l'Association pour les Fouilles Archéologiques Nationales (AFAN) sous la responsabilité scientifique du service régional de l'Archéologie de Guyane avec un financement réalisé majoritairement par EDF mais aussi par l'Etat et les communautés territoriales.

L'aire concernée couvre $310 \mathrm{~km}^{2}$ de forêt primaire équatoriale dont la topographie correspond à un relief accidenté de basse altitude parcouru par un réseau hydrographique très dense.

Lors de la phase de terrain, 311 sites ont été répertoriés dont 273 correspondent à d'anciennes occupations amérindiennes. Parmi celles-ci, 90 sites de plein air ont été identifiés; il s'agit d'anciens villages, de centres artisanaux ou cultuels dont l'implantation géographique est variable. L'occupation amérindienne est attestée avec certitude à partir de 1600 B.P. (Before Present). Un volume scientifique est à paraitre en 1997 et une exposition a eu lieu en Guyane en 1996.

L'autre partie de l'étude a porté sur les implantations coloniales successives, les relations avec les indigènes, la quête incessante de ressources vitales: l'or, la forêt, le sol, voire les hommes et, bien sûr, le souci permanent de domestiquer le fleuve (Puaux et al., 1997).

\section{CONCLUSION}

Au titre de ses obligations réglementaires, EDF doit soumettre, aux Pouvoirs Publics, fin 1998, une synthèse du suivi écologique portant sur les trois premières années de fonctionnement. A ce titre, le suivi continue donc jusqu'en 1998 sur la retenue de Petit-Saut.

II faut souligner que ces études permettront pour la première fois en zone tropicale de "mesurer " l'impact 
d'un barrage sur les différents compartiments de l'écosystème. Les résultats obtenus, même partiels pour certains, sont les seuls à pouvoir apporter des éléments de réponses aux questions relatives au bilan de ce type d'ouvrages.

Enfin, le montant des dépenses consacrées à la protection de l'environnement lors de la réalisation de l'aménagement hydroélectrique de Petit-Saut et lors du suivi écologique évoqué ci-dessus est estimé à $5 \%$ du coût total, soit environ $150 \mathrm{MF}$.

\section{REMERCIEMENTS}

Que soient ici remerciés très sincèrement tous les scientifiques, chercheurs, vétérinaires, archéologues, ingénieurs, techniciens ou stagiaires et tous les "non scientifiques ", ouvriers de capture, piroguiers, layonneurs et autres forestiers, qui ont largement contribué à la bonne réalisation du programme Environnement de Petit-Saut.

Un vif remerciement à tous les membres du Comité Scientifique, à Monsieur Eugène Angelier, à Monsieur Jacques Lecomte, qui ont bien voulu en être les présidents, pour leurs précieux conseils.

\section{BIBLIOGRAPHIE}

Cet article a été rédigé à partir des nombreux documents qui jalonnent le pro- gramme Environnement. II n'est pas possible de les citer tous. Nous citerons seulement :

EDF 1987. Chute de Petit-Saut - Demande de concession et demande de déclaration d'utilité publique - Etude d'impact sur l'environnement.

Dumestre J.F., Labroue L., Galy-Lacaux C., Reynouard C. et Richard S. Revue Hydroécologie Appliquée 1997. Biomasse et activité bactérienne dans la retenue et à l'aval du barrage de PetitSaut (Guyane): influence sur les émissions de méthane et la consommation d'oxygène.

Gosse Ph. et Grégroire A. Revue Hydroécologie Appliquée 1997. Disposition de réoxygénation artificielle de l'eau à l'aval du barrage de Petit-Saut (Guyane).

Puaux O. et Philippe M. 1997. Documents d'Archéologie Française. Editions de la Maison des Sciences de l'Homme. Paris. Archéologie et histoire du Sinnamary du $X V \|^{\theta}$ au $x x^{e} s$. (Guyane).

Richard S., Arnoux A. et Cerdan Ph. Revue Hydroécologie Appliquée 1997. Evolution de la qualité physico-chimique de l'eau de la retenue et du tronçon aval depuis le début de la mise en eau du barrage de Petit-Saut.

Vidal N., De Massary J.C. and Marty C. In press. Quatre nouvelles espèces de serpents pour la Guyane Française. Revue française d'aquariologie.

Vie J.C., Volobouev V., Patton J.L. et Granjon L. 1996. A new species of Isothrix (Rodentia: Echimyidae) from French Guiana. Mammalia 60(3) : 393406. 